Mots. Les langages du politique

$88 \mid 2008$

Discours politique, discours expert

\title{
Quand la Commission européenne promeut la société de la connaissance
}

Roser Cussó

\section{(2) OpenEdition \\ Journals}

Édition électronique

URL : https://journals.openedition.org/mots/14263

DOI : $10.4000 /$ mots. 14263

ISSN : 1960-6001

Éditeur

ENS Éditions

Édition imprimée

Date de publication : 1 novembre 2008

Pagination : 39-52

ISBN : 978-2-84788-147-9

ISSN : 0243-6450

Référence électronique

Roser Cussó, «Quand la Commission européenne promeut la société de la connaissance », Mots. Les langages du politique [En ligne], 88 | 2008, mis en ligne le 01 novembre 2010, consulté le 23 avril 2022. URL : http://journals.openedition.org/mots/14263 ; DOI : https://doi.org/10.4000/mots. 14263 


\section{Quand la Commission européenne promeut la «société de la connaissance»}

La Commission européenne définit la «société de la connaissance» comme étant la «société dont les processus et les pratiques sont fondés sur la production, la diffusion et l'utilisation des connaissances »'1. Elle serait liée au développement des nouvelles technologies, mais surtout aux «échanges, [aux] déplacements et [aux] communications à l'échelle planétaire [qui] élargissent l'horizon culturel de chacun d'entre nous et bouleversent les règles de concurrence entre les économies $»^{2}$. Pour la Commission européenne, «l'Europe d'aujourd'hui connaît une transformation d'une ampleur comparable à celle de la révolution industrielle $» 3$.

La notion de société de la connaissance est directement liée à une interprétation globale des évolutions socio-économiques récentes. Trois autres dimensions centrales viennent la compléter: une politique économique, une perception de ce que l'éducation et la formation devraient devenir, et la définition d'un nouvel espace public.

L'analyse du contenu et l'analyse lexicométrique4 des principaux documents officiels de la Commission européenne se référant à l'éducation et à la formation (1995-2003) ont pour but, dans cet article, de préciser les caractéristiques de ces quatre dimensions, résumées dans les termes mondialisation, compétitivité, réforme et partenariat. Il s'agit d'un Livre blanc, d'un document de travail de la Commission et de six propositions ou autres communications de cette dernière au Conseil et/ou aux autres institutions (voir le tableau 1 pour les références complètes). Le Livre blanc sur l'éducation et la formation est le premier texte où la société de la connaissance apparait comme l'objectif principal des propositions européennes, ces dernières étant destinées à encourager la réforme des systèmes éducatifs des États membres.

Université Paris 8, LDH (EHESS), rosercusso@hotmail.com

1. COM (2001) 678. Toutes les citations contenues dans cet article proviennent d'un même corpus de textes de la Commission européenne (1995-2003) dont le détail est présenté dans le tableau 1.

2. $\operatorname{COM}(2001) 59$.

3. SEC (2000) 183.

4. Le logiciel d'analyse statistique lexicale utilisé est Lexico3, développé par le SYLED, Université Paris 3, sous la direction d'André Salem. 
Les autres textes font suite à ces propositions. Deux documents visent à les préciser et/ou les compléter; trois autres cherchent à traduire ces propositions en objectifs communs concrets. Enfin, les deux derniers visent respectivement à encourager les investissements (privés) dans l'éducation et à évaluer les progrès des États membres vers la réalisation des objectifs communs. Le tableau 2 synthétise les principales caractéristiques du corpus de textes analysé dans cet article.

Tableau 1. Présentation du corpus (1995-2003)

\begin{tabular}{|l|l|c|}
\hline CE* $1995^{*}$ & $\begin{array}{l}\text { Livre blanc sur l'éducation et la formation. Enseigner } \\
\text { et apprendre. Vers la société cognitive }\end{array}$ & COM (1995) 590 \\
\hline CE 2000 & $\begin{array}{l}\text { Mémorandum sur l'éducation et la formation tout } \\
\text { au long de la vie. Document de travail des services } \\
\text { de la commission des Communautés européennes }\end{array}$ & SEC (2000) 1832 \\
\hline CE 2001 & Les objectifs concrets futurs des systèmes d'éducation & COM (2001) 59 \\
\hline CE 2001 & $\begin{array}{l}\text { Projet de programme de travail détaillé sur le suivi } \\
\text { du rapport concernant les objectifs concrets } \\
\text { des systèmes d'éducation et de formation }\end{array}$ & COM (2001) 501 \\
\hline CE 2001 & $\begin{array}{l}\text { Réaliser un espace européen de l'éducation } \\
\text { et de la formation tout au long de la vie }\end{array}$ & COM (2001) 678 \\
\hline CE 2002 & $\begin{array}{l}\text { Critères de référence européens pour l'éducation } \\
\text { et la formation: suivi du Conseil européen de Lisbonne }\end{array}$ & COM (2002) 629 \\
\hline CE 2003 & $\begin{array}{l}\text { Investir efficacement dans l'éducation } \\
\text { et la formation: un impératif pour l'Europe }\end{array}$ & COM (2002) 779 \\
\hline CE 2003 & $\begin{array}{l}\text { Éducation + formation 2010. L'urgence des réformes } \\
\text { pour réussir la stratégie de Lisbonne }\end{array}$ & COM (2003) 685 \\
\hline
\end{tabular}

*Commission européenne.

Tableau 2. Principales caractéristiques du corpus

\begin{tabular}{lcc}
\hline \multicolumn{1}{c}{ Partie } & $\begin{array}{c}\text { Nombre } \\
\text { d'occurrences }\end{array}$ & $\begin{array}{c}\text { Nombre } \\
\text { de formes }\end{array}$ \\
\hline $\operatorname{COM}(1995) 590$ & 27231 & 3724 \\
$\operatorname{SEC}(2000) 1832$ & 20324 & 2884 \\
$\operatorname{COM}(2001) 59$ & 12444 & 2131 \\
$\operatorname{COM}(2001) 501$ & 8469 & 1571 \\
$\operatorname{COM}(2001) 678$ & 19301 & 2089 \\
$\operatorname{COM}(2002) 629$ & 7323 & 1280 \\
$\operatorname{COM}(2002) 779$ & 14306 & 2125 \\
$\operatorname{COM}(2003) 685$ & 8790 & 1552 \\
Total & 118188 & 17356 \\
\hline
\end{tabular}


Cette analyse permettra, dans un premier temps, de mieux comprendre le caractère programmatique et l'articulation entre les interprétations, les conclusions et les propositions de la Commission : elles apparaissent comme étant à la fois interdépendantes et nécessaires. En deuxième lieu, nous analyserons plus amplement la perception des politiques publiques soutenues par la Commission européenne. Cette vision illustre la consolidation d'un nouveau référentiel politique (Van Haecht, 1997) : celui qui octroie à l'action publique un rôle régulateur du lien entre la société civile et l'économie plutôt qu'un rôle redistributeur à l'intérieur d'une société politique liée à la sphère économique par une relation plus conflictuelle. Les grands traits de ce type de transformation dans le langage politique avaient déjà été ébauchés par Corinne Gobin et Jean-Claude Deroubaix dans le contexte des déclarations gouvernementales belges entre 1944 et 1988 (Gobin, Deroubaix, 1989). Nous nous centrerons ici sur les caractéristiques lexicales et argumentatives s'inscrivant dans ce qui semble être l'aboutissement d'une telle transformation dans le cadre de l'Union européenne (UE).

Ainsi, la fréquence relative de certains termes et de certaines expressions dans les textes de la Commission européenne ainsi que l'analyse de leur contenu et de leurs contextes permettent non seulement d'illustrer l'importance centrale de la société de la connaissance comme paradigme socioéconomique (nouveaux problèmes et nouvelles solutions auxquels les acteurs sont encouragés à faire référence), mais aussi de souligner son articulation avec les termes et expressions apprentissage, travail, emploi, compétences ou formation tout au long de la vie (voir le tableau 2 ci-contre5). En complément à l'analyse de cette orientation, centrée sur l'adaptation des systèmes éducatifs au marché de l'emploi (Cussó, 2007), nous pouvons également faire remarquer l'absence d'expressions telles que droit ou négociation collective (Cussó, 2005), qui s'avéreraient plus cohérents avec une perception socialedémocrate de la pratique politique, de l'espace public et des systèmes démocratiques en général (Gobin, 2002).

Enfin, l'analyse du contenu et l'analyse lexicométrique des textes communautaires permettent de compléter l'étude du rôle des statistiques dans la méthode ouverte de coordination ou MOC (Cussó, 2004). Lexique et statistique, interprétation et proposition pratique sont des aspects complémentaires dans l'activité politique et idéologique de la Commission européenne dans le domaine de l'éducation.

5. «[L']utilisation d'un lexique particulier et la répétition plus ou moins grande de certaines formes de ce lexique sont des caractéristiques importantes de tout discours politique. Le lexique et les fréquences d'emploi de formes apparaissent comme les signatures du discours [...] » (Gobin, Deroubaix, 1989, p. 147). 


\section{Autonomie de la Commission européenne et investissement du domaine de l'éducation}

La Commission européenne a un rôle très important d'initiative politique au sein de l'Union européenne, ainsi qu'une forte autonomie par rapport aux autres institutions européennes. D’une part, la Commission propose la législation, les politiques et les programmes d'action pour l'Europe (par la production de directives ou à travers la coordination politique). Elle est également responsable de la mise en œuvre des décisions du Parlement et du Conseil européen. D'autre part, tandis que la Commission s'appuie sur son travail d'expertise, son contrôle politique est plutôt faible du fait de la structure même de l'Union. Présentée comme étant une «institution politiquement indépendante ${ }^{6}$, la Commission n'est pas soumise au contrôle qui serait exercé, en principe, par les partis politiques sur un gouvernement de la majorité.

En contraste avec d'autres domaines, l'Union européenne n'a pas de compétences directes dans le domaine de l'éducation. Toutefois, en s'appuyant sur une interprétation large de la notion de formation professionnelle (Cussó, 2006), la Commission européenne a progressivement investi le champ éducatif. C'est avec le Traité de Maastricht (1992) que la compétence de l'UE en matière d'enseignement est officiellement reconnue et, en même temps, encadrée par le principe de subsidiarité. La Commission ne peut donc pas, pour le moment, procéder par directives dans ce domaine. Elle peut toutefois recourir à des recommandations et à des mesures d'encouragement. C'est dans ce cadre qu'un processus de coordination politique a été entamé, fruit notamment de l'interaction entre la Commission et le Conseil. La définition de la stratégie de Lisbonne (construction d'une société européenne de la connaissance7) en 2000, l'accord autour des objectifs communs pour l'éducation et la formation en 2001 et l'adoption de la MOC pour les piloter en 2002, témoignent de la mise en œuvre de ce processus de concertation. Le corpus ici analysé contient une partie importante des documents soutenant cette nouvelle coordination politique pour l'enseignement et la formation.

Comme signalé plus haut, l'émergence de la notion de société de la connaissance $^{8}$ dans les textes de la Commission européenne accompagne, tout d'abord, une interprétation large des évolutions économiques et techno-

6. Union européenne, 2003, Comment fonctionne l'Union européenne?, Bruxelles, p. 20.

7. La stratégie de Lisbonne est un ensemble d'orientations politiques prises lors du sommet européen de mars 2000, réunissant les chefs d'État et de gouvernement de l'Union. On peut en trouver les détails dans les « Conclusions de la Présidence. Conseil européen de Lisbonne, 23 et 24 mars 2000 », DOC/00/8, 2000. Lien: http://ue.eu.int/ueDocs/cms_Data/docs/pressData/fr/ ec/o0100-r1.fo.htm

8. Pour un historique de l'origine et de l'évolution de cette expression, voir Ranson (1998), Faure (1972) et NCEE (1983). 
logiques récentes: «Dans le monde entier, les technologies de l'information et des télécommunications engendrent une nouvelle révolution industrielle, qui apparaît d'ores et déjà aussi importante et radicale que celles qui l'ont précédée. »9 Les facteurs du changement et leurs conséquences sur l'éducation seraient multiples:

En cette fin de siècle, les causes de changement de la société ont été diverses et toutes ont des conséquences, à un degré ou à un autre, sur les systèmes d'éducation et de formation. L'évolution démographique a accru la durée de vie en même temps qu'elle a profondément modifié la pyramide des âges [...]. L'augmentation considérable du nombre de femmes exerçant un emploi a modifié la place traditionnelle de la famille [...]. Les innovations technologiques se sont multipliées dans tous les domaines [...]. Les modes de consommation ont évolué, de même que les styles de vie. Également, une prise de conscience s'étend sur les problèmes d'environnement et l'utilisation des ressources naturelles [.... $]^{10}$.

Parmi ces facteurs, la Commission européenne retient «trois chocs moteurs» qui « transforment profondément et durablement le contexte de l'activité économique et le fonctionnement de nos sociétés. Ce sont l'avènement de la société de l'information ainsi que le développement de la civilisation scientifique et technique et la mondialisation de l'économie». Parmi ces «chocs moteurs », la mondialisation est particulièrement importante: «La mondialisation de l'économie [...] s'est traduite par une libre circulation sans précédent des capitaux, des biens et des services. Demain se créera, plus vite que l'on ne le croit généralement, un marché global et différencié de l'emploi. »11

Tandis que la libre circulation des capitaux n'est plus analysée (et encore moins problématisée), l'attention de la Commission européenne se centre sur le marché global de l'emploi qui devrait être créé « plus vite que l'on ne le croit». Les termes capital/aux sont, en effet, relativement peu nombreux. Ils comptent ensemble 27 occurrences, dont 18 se réfèrent au capital humain. Seules 7 occurrences concernent le capital économique (son marché, sa circulation, sa dimension financière). Les conditions politiques de sa détaxation sont complètement absentes. En contraste, les expressions marché du travail et marché de l'emploi sont plus nombreuses (65 occurrences) ainsi que marché/s (105 occurrences). En fait, travail et emploi comptent ensemble plus de 500 occurrences. Ces deux termes apparaissent en $13^{\mathrm{e}}$ et $17^{\mathrm{e}}$ position respectivement parmi les 40 premières occurrences du corpus - comprenant uniquement les substantifs, les adjectifs et les verbes autres qu'être et avoir (voir le tableau 3 page suivante).

9. $\operatorname{COM}(1995) 590$.

10. Ibid.

11. Ibid. 
Tableau 3. Les premières 40 occurrences du corpus

\begin{tabular}{clc}
\hline Rang & Occurrences & Fréquences \\
\hline 1 & formation & 1396 \\
2 & éducation & 1240 \\
3 & tout & 536 \\
4 & vie & 533 \\
5 & long & 444 \\
6 & États & 388 \\
7 & apprentissage & 375 \\
8 & européen & 370 \\
9 & compétences & 349 \\
10 & membres & 345 \\
11 & niveau & 304 \\
12 & enseignement & 303 \\
13 & travail & 273 \\
14 & société & 271 \\
15 & conseil & 255 \\
16 & commission & 249 \\
17 & emploi & 237 \\
18 & systèmes & 237 \\
19 & pays & 229 \\
20 & européenne & 228 \\
\hline
\end{tabular}

\begin{tabular}{clc}
\hline Rang & Occurrences & Fréquences \\
\hline 21 & objectifs & 219 \\
22 & Europe & 207 \\
23 & cadre & 204 \\
24 & qualité & 202 \\
25 & jeunes & 179 \\
26 & développement & 178 \\
27 & ressources & 178 \\
28 & base & 174 \\
29 & mise & 173 \\
30 & domaine & 163 \\
31 & union & 162 \\
32 & autres & 159 \\
33 & entreprises & 158 \\
34 & connaissance & 156 \\
35 & investissement & 154 \\
36 & Lisbonne & 154 \\
37 & rapport & 153 \\
38 & niveaux & 141 \\
39 & œuvre & 140 \\
40 & domaines & 135 \\
\hline
\end{tabular}

\section{Politique économique : organiser l'ouverture, augmenter la compétitivité}

À partir de son interprétation univoque des évolutions sociales et économiques (notamment l'émergence de la mondialisation), la Commission européenne fait un choix clair: "Dans son Livre blanc "Croissance, Compétitivité, Emploi", la Commission a clairement fait le pari de l'ouverture surle monde [...]. Elle a en particulier mis l'accent sur l'importance du maintien du modèle social européen. » Dans ce sens, la Commission européenne soutient que «le choix de l'ouverture, qui pousse à développer la compétitivité générale de nos économies, augmente le bien-être général en rendant plus efficace l'allocation mondiale de ressources». Par ailleurs, «la mondialisation ne fait [...] que renforcer la pertinence de l'Europe comme niveau d'intervention. Dans un monde mouvant et incertain, l'Europe est un facteur d'organisation $»^{12}$.

Ce qui est central est d'aider l'Union européenne à devenir «l'économie de la connaissance la plus compétitive et la plus dynamique du monde». L'encouragement à une plus grande compétitivité est souvent le pivot des pas-

12. Ibid. 
sages à teneur politique: "L'Union européenne, premier exportateur mondial, a choisi très logiquement l'ouverture sur l'économie mondiale : il lui faut donc, en permanence, renforcer sa compétitivité économique. $»^{13}$ Ou encore: «La compétitivité et le dynamisme sont deux aspects sur lesquels l'UE accuse actuellement du retard sur les États-Unis. $»^{14}$

L'augmentation de la compétitivité européenne dépendrait principalement de la satisfaction des nouveaux besoins du marché de l'emploi. Ces besoins concernent, entre autres, le développement des compétences et leur mise à jour continue. Il est ainsi noté que «l'investissement dans les compétences est reconnu comme le facteur central de la compétitivité et de l'aptitude à l'emploi par le Livre blanc ${ }^{25}$. En fait, compétence est, avec les termes emploi et travail, un des plus fréquents du corpus, référant à une notion clé, avec 349 occurrences (voir le tableau 3).

Enfin, une fois établi le lien entre les compétences et les besoins du marché de l'emploi (par le biais de la compétitivité), la transformation des systèmes éducatifs apparait comme étant nécessaire: «Relever le niveau de l'éducation et de la formation est essentiel pour renforcer la compétitivité et le dynamisme de l'Europe. ${ }^{16}$

\section{Réforme des systèmes éducatifs : adaptation, flexibilité, harmonisation}

"Les Chefs d'État et de gouvernement réunis pour les Conseils européens de printemps de Lisbonne (en 2000), Stockholm (en 2001) et Barcelone (en 2002), ont confirmé le rôle et l'importance de l'éducation et de la formation et ont fixé des priorités pour une action concertée au niveau européen. ${ }^{17} \|$ s'agit de développer « un programme ambitieux en vue de moderniser les systèmes de sécurité sociale et d'éducation». Dans ce sens, «la transition vers une société et une économie de la connaissance et les nouveaux besoins en matière d'apprentissage tout au long de la vie appellent des changements radicaux des systèmes d'éducation et de formation ${ }^{18}$.

Les termes du corpus exprimant le changement (adaptation, transformation, modernisation...) dépassent les 280 occurrences (voir le tableau 4 page suivante).

\footnotetext{
13. Ibid.

14. $\operatorname{COM}(2002) 779$.

15. COM (1995) 590.

16. $\operatorname{COM}(2001) 59$.

17. $\operatorname{COM}(2002) 779$.

18. $\operatorname{COM}(2003) 685$.
} 
Tableau 4. Formes lexicales liées à la réforme

\begin{tabular}{cc}
\hline Formes lexicales & Fréquences \\
\hline adapt- & 109 \\
chang- & 61 \\
réform- & 58 \\
transform- & 31 \\
modern- & 22 \\
\hline
\end{tabular}

Au-delà de la fréquence relative des différentes occurrences, l'analyse du contenu de leurs contextes permet de noter le fait que le pont entre la réforme des systèmes éducatifs et l'emploi est systématiquement établi dans un sens précis: l'éducation doit s'adapter aux besoins du marché du travail. En effet, dans la presque totalité des contextes de marché de l'emploi et de marché du travail - où le domaine de l'enseignement est cité -, le lien entre l'éducation et le marché apparait en termes d'adaptation (ou de rapprochement) de la première au deuxième (qu'il s'agisse de l'organisation, du contenu, des qualifications). Les propositions et/ou explications relatives à cette adaptation sont explicites: «Les systèmes d'enseignement et de formation professionnels jouent un rôle essentiel afin d'équiper les individus de compétences et de qualifications qui répondent aux besoins du marché du travail. [...] Pour être compétitive dans l'économie de la connaissance, l'Union a aussi besoin d'un nombre suffisant de diplômés de l'enseignement supérieur disposant d'une préparation adaptée au marché du travail européen. ${ }^{19}$

Toujours dans la logique du lien entre l’éducation et le marché (européen) de l'emploi, il s'agira également de promouvoir un certain rapprochement, voire une harmonisation des systèmes éducatifs et des systèmes de formation nationaux:

La globalisation implique non seulement des investissements plus élevés, mais aussi un processus parallèle de réformes pour améliorer la qualité et la pertinence des programmes [...] et la cohérence des systèmes d'éducation et de formation en Europe et pour accroître leur visibilité et leur reconnaissance ailleurs dans le monde. [...] Le développement du processus de Bologne dans l'enseignement supérieur et de celui de Bruges dans la formation professionnelle constitue des pas dans la bonne direction ${ }^{20}$.

Enfin, la réforme des systèmes éducatifs est également liée à la flexibilité dans le sens de la différentiation de l'offre scolaire selon les publics: «La question

19. Ibid.

20. COM (2002) 779. 
centrale est d'aller vers une plus grande flexibilité de l'éducation et de la formation, permettant de prendre en compte la diversité des publics et des demandes. $»^{21}$

\section{Redéfinition de l'espace public : accord entre partenaires}

Dans le projet de réforme des systèmes éducatifs proposé par la Commission, le rôle des acteurs serait central:

Les stratégies d'éducation et de formation tout au long de la vie doivent rassembler tous les acteurs concernés et être soutenues par eux; [...] les États membres devraient associer tous les acteurs, y compris ceux offrant un apprentissage non formel, les partenaires sociaux, les représentants du secteur de l'apprentissage formel (enseignement professionnel et supérieur) et les ONG représentant des groupes exclus, à l'établissement de méthodologies et de normes de valorisation de l'apprentissage non formel et informel22.

Toutefois, si les acteurs sont souvent évoqués, ils ne constituent pas tout à fait des acteurs politiques (Gobin, 2005). Par exemple, les formes syndicats et syndicales n'apparaissent que trois fois dans tout le corpus; partis (politiques), une seule fois. Ces institutions ne sont citées que dans le contexte de l'offre d'éducation non formelle qu'elles produisent: "L'éducation non formelle peut s'acquérir sur le lieu de travail ou dans le cadre des activités d'organisations ou de groupes de la société civile (associations de jeunes, syndi-

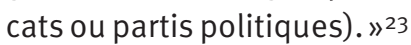

En fait, avec les associations d'employeurs, les syndicats sont intégrés dans l'expression plus générale partenaires sociaux (56 occurrences). Ces partenaires sociaux coopèrent (plutôt qu'ils ne se confrontent) y compris dans des domaines particulièrement sensibles: «Rapprocher l'école et l'entreprise est donc une priorité, à laquelle les partenaires sociaux doivent prendre toute leur part. » 24 Cette coopération prend souvent la forme plus large de «partenariat/s» (65 occurrences du mot) : «Un autre aspect important concerne l'implication de tous les acteurs utiles dans le cadre d'une approche de partenariat. » Dans ce contexte, l'ensemble de la société est supposé appuyer un même projet: «Tous les acteurs sont responsables de coopérer dans le domaine de l'éducation et de la formation tout au long de la vie [...]»:

Les employeurs sont principalement responsables du développement des compétences de leurs travailleurs et assument de plus des responsabilités sociales plus

\footnotetext{
21. $\operatorname{COM}(1995) 590$.

22. $\operatorname{COM~(2001)~} 678$.

23. SEC (2000) 1832.

24. COM (1995) 590.
} 
larges [...]. Les groupements bénévoles et locaux sont à même de proposer des offres ciblées d'éducation et de formation [... $]^{25}$.

Cette perception des acteurs est en liaison avec l'introduction de la notion de gouvernance au sein de l'activité de la Commission. Celle-ci est directement liée au développement des consultations:

[...] la Commission européenne a présenté un Mémorandum sur l'éducation et la formation tout au long de la vie. Ce dernier a servi de base à une consultation à l'échelle européenne, organisée aussi près des citoyens que possible, conformément à l'objectif de la Commission de réformer la gouvernance européenne ${ }^{26}$.

Plus précisément, cette «gouvernance européenne» serait l'«Administration des affaires européennes passant par l'interaction des autorités politiques traditionnelles et de la "société civile", des acteurs du secteur privé, des organisations publiques et des citoyens $\gg 27$. Les autorités politiques traditionnelles seraient-elles les élus et, en général, les représentants politiques des citoyens? Si c'est le cas, leur relation avec ces derniers pourrait-elle relever d'une «interaction » ? À travers la prédominance de la gouvernance, l'espace public - et par là, le débat politique - est redéfini, tout au moins en principe.

Parmi les quatre dimensions fondant les textes de la Commission européenne, la dernière (partenariat) est celle qui touche le plus directement la transformation (ou la tentative de transformation) du référentiel politique européen, celui des «politiques traditionnelles» qui auraient eu «tendance à trop insister sur les dispositifs institutionnels [...] $»^{28}$. Selon la Commission européenne, "de nouvelles conceptions de l'équilibre entre les droits et les responsabilités des citoyens et des pouvoirs publics ont commencé à s'affirmer». Par exemple:

La plus grande liberté dont jouissent les directeurs [d'établissement] leur permet de conclure un type de partenariat différent avec les pouvoirs publics, qui ne soit pas uniquement bilatéral mais multilatéral et englobe non seulement d'autres acteurs du monde de l'éducation et de la formation [...], mais également des organismes privés tels que les entreprises 29 .

On remarque ici que les pouvoirs publics seraient, en définitive, des acteurs parmi d'autres. Ainsi: «Un accroissement réel et durable de l'investissement dans les ressources humaines demande une action de la part de tous les acteurs concernés: individus, entreprises, partenaires sociaux et pouvoirs

\footnotetext{
25. $\operatorname{COM}(2001) 678$.

26. Ibid.

27. Ibid.

28. Ibid.

29. $\operatorname{COM}(2001) 59$.
} 
publics. „30 Les extraits ci-dessous présentent les autres contextes de la lexie pouvoirs publics dans le corpus [nous soulignons] :

[...] dans ces domaines [l'enseignement supérieur, la formation des adultes et la formation professionnelle continue] où les pouvoirs publics doivent conserver tout leur rôle, la contribution privée paraît néanmoins plus indispensable [...] et plus justifiée que dans l'enseignement obligatoire [...]

Cet objectif [...] se réfère à l'ensemble des investissements, c'est-à-dire à ceux des pouvoirs publics, des entreprises privées et des particuliers.

Les pouvoirs publics portent la responsabilité de s'attaquer à ces déficits de financement [...]. Mais ils ne peuvent y réussir sans le soutien d'une grande variété de partenaires.

[...] la principale responsabilité des pouvoirs publics est non seulement de continuer à fournir aux établissements d'enseignement supérieur et aux étudiants un niveau suffisant de financement public, mais aussi de trouver des moyens de l'augmenter en accroissant et en diversifiant les participations privées [...]

Selon l'OCDE, une convergence de vues est apparue vers la fin des années 1990 pour reconnaître que les pouvoirs publics seuls ne pouvaient pas fournir l'ensemble des ressources nécessaires à l'apprentissage tout au long de la vie [...]

Les pouvoirs publics des États membres et des Pays Candidats portent la responsabilité de réaliser une «augmentation substantielle» de l'investissement total.

Y parvenir exigera une approche de partenariat et une action de toutes les parties intéressées: individus, entreprises, partenaires sociaux et pouvoirs publics.

Société civile: «Troisième secteur» de la société, distinct de l’État et du marché, constitué d'institutions, de groupes et d'associations (structurés ou non) susceptibles d'exercer une fonction de médiation entre les pouvoirs publics et les citoyens.

[Indicateur sur l']Aide accordée par les pouvoirs publics dans le cadre de la mise sur pied de systèmes d'amélioration et d'assurance de la qualité [...]

Ces partenariats incluent une coopération entre les ministères et les pouvoirs publics en vue de définir des politiques coordonnées. Ils intègrent systématiquement les partenaires sociaux au processus d'élaboration et de mise en œuvre des politiques, en liaison avec des initiatives publiques/privées.

La promotion de la culture scientifique et technique fait l'objet d'une attention soutenue des pouvoirs publics.

Aujourd'hui, il s'agit [...] de tenter de mettre d'accord les acteurs: les formateurs, les entreprises, les pouvoirs publics sur des orientations nouvelles qui pourraient rapidement prendre la forme de mesures concrètes.

30. $\operatorname{COM}(2002) 779$. 
Dans un contexte totalement différent, mais également pour répondre à une situation de crise, il n'est pas étonnant qu'en Europe des pouvoirs publics locaux, s'appuyant sur des associations, renouent avec l'idée, lancée initialement en Europe dès l'entre-deux-guerres, d'offrir par l'école une deuxième chance.

Pour la Commission européenne, les pouvoirs publics font ainsi partie d'un ensemble d'acteurs dont le degré de responsabilité et de légitimité politiques est souvent difficile à définir31. Devant, en principe, contribuer activement à la mise en œuvre de la gouvernance et du partenariat, les pouvoirs publics seraient un des secteurs de la société, avec le marché et la société civile. En fait, c'est cette dernière qui serait la plus apte à «exercer une fonction de médiation entre les pouvoirs publics et les citoyens ${ }^{32}$. Les pouvoirs publics sont, enfin, supposés encourager l'investissement privé dans l'enseignement et réguler l'assurance de la qualité de l'éducation en général.

Il est possible de lire les textes du corpus ici analysé selon quatre dimensions qui s'alignent aussi en quatre temps d'argumentation: (i) les évolutions socio-économiques récentes (libre circulation des capitaux, mondialisation) étant considérées comme des variables exogènes (société de la connaissance), la Commission européenne rejoint (ii) le pari de «l'ouverture sur le monde». Dans ce contexte, la compétitivité de l'UE doit être renforcée, voire augmentée. Et elle nécessite l'encouragement et l'organisation d'un marché global de l'emploi. Ce dernier dépend, à son tour, de (iii) la réforme approfondie des systèmes éducatifs et de formation. Cette réforme devrait se centrer sur la flexibilité, la compatibilité européenne des qualifications et des systèmes (pour faciliter la mobilité de la main-d'œuvre), le développement des compétences des élèves et des étudiants et l'augmentation des investissements privés. Ce processus s'accompagne, enfin, de (iv) la définition d'un nouvel espace public: il se caractérise par la coopération entre partenaires et acteurs partageant les mêmes objectifs (partenariat). Cette coopération est, entre autres, le résultat de l'interaction entre les pouvoirs publics, la société civile et le marché, chacun représentant un secteur de la société (gouvernance).

L'expression société de la connaissance prend le pas sur capitalisme cognitif et mobilité des capitaux ou sur d'autres formules qui pourraient donner une dimension plus normative aux évolutions récentes dans le domaine de l'économie. L'expression société de la connaissance, telle qu'utilisée, permet une perception plus neutre des choix de la Commission européenne qui restent toutefois idéologiquement très marqués.

31. Les termes ou expressions élu(s), élection(s), responsable(s) politique(s), représentant(s) politique(s) ne sont jamais cités dans le corpus.

32. $\operatorname{COM}(2001) 678$. 
La tonalité de ces choix est mise en évidence par le type de réforme des systèmes éducatifs proposée par la Commission européenne. Bien que des termes tels que ouverture, flexibilité ou éducation et formation tout au long de la vie semblent octroyer un cadre plus autonome et souple à l'apprentissage et s'insérer même dans une vision progressiste de l'enseignement33, ce projet éducatif reste attaché à une priorité sans faille pour l'adaptation de l'éducation aux besoins du marché de l'emploi par le développement des compétences, mais aussi par le biais de l'augmentation des investissements privés.

La vision de l'enseignement défendue par la Commission européenne ne s'arrête pas à la promotion de cette adaptation. La réforme des systèmes éducatifs des États membres est attachée à une certaine simplification de l'espace public (notamment de sa dimension conflictuelle) et suppose implicitement une mutation profonde de la perception des politiques publiques. La proposition de réforme des systèmes d'éducation introduit, en fait, une nouvelle vision du fonctionnement démocratique dans son ensemble. L'idée de pluralité dans l'interprétation des évolutions sociales et économiques contemporaines et dans la manière dont le débat public peut être développé est absente. De même, l'existence de propositions politiques divergentes autour de l'avenir de l'enseignement est omise. Consultation et partenariat surplombent le débat politique.

Enfin, l'interdépendance entre l'interprétation des évolutions sociales récentes et les propositions de réforme de la Commission européenne donne lieu à un discours politique global et circulaire. Les quatre dimensions analysées plus haut constituent une chaine cohérente d’idées aboutissant à une seule conclusion possible, tout au moins en principe, celle d'une réforme précise de l'enseignement. La Commission européenne semble donc produire un programme politique assimilable à celui que produirait une pensée politique particulière. Elle est pour autant présentée comme étant une institution politiquement indépendante. Plusieurs questions restent ainsi ouvertes: Quelle est la place politique des propositions de la Commission européenne? Quelle est la possibilité de présenter un projet alternatif et dans quel espace politique européen?

\section{Références}

Cussó Roser, 2004, "La méthode ouverte de coordination en Europe: des statistiques pour une nouvelle politique d'éducation», Séminaire RAPPE. Les comparaisons nationales et internationales des politiques éducatives, 24-25 mai 2004, Paris.

33. Dans la tradition de la critique libertaire des systèmes éducatifs formels, voir par exemple Illich (1971). 
- 2005, "La rhétorique de la société de la connaissance et l’Europe: vers un rétrécissement de l'espace public », Revue de l'Institut de sociologie, n 1-2, p. 75-92.

- 2006, "La Commission européenne et l'enseignement supérieur: une réforme au-delà de Bologne », Cahiers de la recherche sur l'éducation et les savoirs, $\mathrm{n}^{\circ} 5$, septembre.

— 2007, «Malgré les consultations: vers une adaptation accrue de l'enseignement au marché du travail en Europe », Vivre ensemble au xxı siècle. Actes du colloque de l'Institut de sociologie, 6-7 octobre 2005, Bruxelles, ULB, p. 137-151.

FAURE Edgar éd., 1972, Apprendre à être, Paris, Unesco-Fayard.

Gobin Corinne, Deroubaix Jean-Claude, 1989, "Les temps sociaux et le discours politique. Repérages de la notion de temps dans les déclarations gouvernementales belges ", Histoire et mesure, t. IV, $\mathrm{n}^{\circ} 1 / 2$, p. 147-171.

- 2002, "Le discours programmatique de l'Union européenne: d'une privatisation de l'économie à une privatisation du politique? ", Sciences de la société, $\mathrm{n}^{\circ} 55$, p. 156-169.

— 2005, "L'Union européenne : où est passé l'acteur? ", Cahiers lillois d'économie et de sociologie, p. 65-88.

ILliCH Ivan D., 1971, Libérer l'avenir. Appel à une révolution des institutions, Paris, Le Seuil.

NCEE (National Council on Economic Education), 1983, A Nation a Risk: the Imperative for Educational Reform, Washington, US Department of Education.

RANSON Stewart éd., 1998, Inside the Learning Society, Londres, Cassell.

VAN HAECHT A., 1997, "Les politiques éducatives, figure exemplaire des politiques publiques? ", Éducation et sociétés, n 1, p. 21-46. 